\title{
Time calibration of the LHAASO-WCDA detectors
}

\section{Bo Gao,,${ }^{a *}$ Jinyan Liu, ${ }^{a}$ Lu Zhang, ${ }^{b}$ Mingjun Chen, ${ }^{a}$ Min Zha, ${ }^{a}$ Zhiguo Yao ${ }^{a}$ and for the LHAASO collaboration ${ }^{a}$}

${ }^{a}$ Institution of High Energy Physics, CAS

19B Yuquan Road, Shijingshan District, Beijing, China

${ }^{b}$ Hebei Normal University, College of Physics \& Information Engineering

No.20, Road E. 2nd Ring South, Yuhua District, Shijiazhuang City, Hebei Province, China

E-mail: gaobo@ihep.ac.cn, liujy@ihep.ac.cn

The LHAASO (Large High Altitude Air Shower Observatory) is a multi-purpose experiment for measuring the high energy gamma rays and cosmic rays. One of the major detectors is the 78,000 $m^{2}$ WCDA (Water Cherenkov Detector Array), equipped with 3120 PMTs, which aims to survey the gamma-ray sky continuously in a wide energy range, from $100 \mathrm{GeV}$ to $30 \mathrm{TeV}$. Precisely calibrating the time offsets of each detector cell is essential to obtain a good angular resolution for observing the gamma ray sources[1]. A dedicated system composed of LED light sources and fibers guided lights to every cell is used for time offset calibration of the whole array. Besides, Cosmic-ray shower events are analyzied for caliculating the time offsets and the charge-time correlations. Finally the observation to the Crab Nebula is visited to fix the pointing error brought by above calibration and calculations. Above calibration procedure and the final calibration results are presented in this talk.

$37^{\text {th }}$ International Cosmic Ray Conference (ICRC 2021)

July 12th - 23rd, 2021

Online - Berlin, Germany

\footnotetext{
*Presenter
} 


\section{Introduction}

LHAASO-WCDA is located at Haizi Mountain, Daocheng, Sichuan Province, China, $4410 \mathrm{~m}$ a.s.l. It is a ground-based cosmic ray detector array using water Cherenkov technique, covers an area of 78,000 $\mathrm{m}^{2}$ and is composed of three pools: pool 1\#2\# covers an area of $150 \mathrm{~m} \times 150 \mathrm{~m}$ for each, pool 3\# covers an area of $300 \mathrm{~m} \times 110 \mathrm{~m}$. The whole WCDA is divided into 3120 detector units (Fig 1). Four types of PMTs are applied. The pool 1\# uses an 8-inch CR365 and a 1.5 inch XP3960 PMT, and the pool 2\#3\# use 20-inch MCP-PMT and 3.5 inch XP72B20 dynode PMT. Among them, two large-size PMTs are mainly used for time measurement, and two small-size PMTs are mainly used for dynamic range expansion. In order to accurately reconstruct the direction of the cosmic ray shower, the time calibration accuracy needs to be better than $0.1 \mathrm{~ns}$.

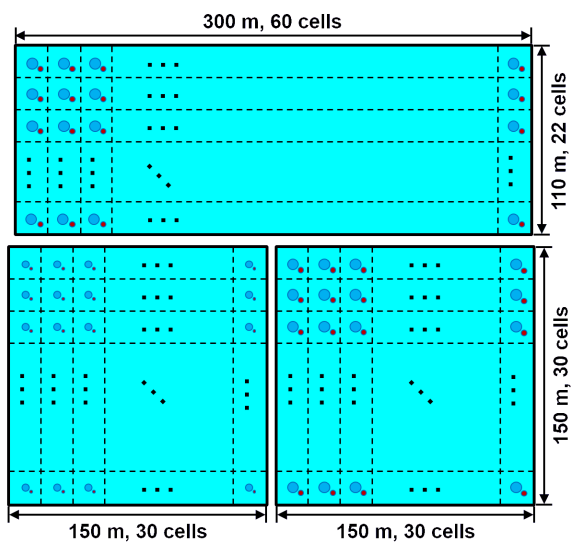

Figure 1: LHAASO-WCDA detector layout.

\section{Time calibration by hardware}

\subsection{Calibration method}

In order to achieve the time calibration of such a large area detector, the hardware calibration system is designed as a distributed cross calibration scheme based on electronic cluster, which can reduce the calibration light pulse transmission length and attenuation. Each cluster consists of 36 channels, two identical time calibration modules (LED 1 and LED 2) are installed, each module contains a blue LEDs and a plastic fiber bundle which transmit the calibration light pulse to PMT, the 2 LEDs are driven by a 2-channel LED driver. LED 1 is used for calibrating 36 detector channels inside the cluster; for LED 2, 20 fibers for the internal calibration of the cluster, and 4 and adjacent cluster 4 detector channels are exchanged for cross calibration. In this way, the calibration of the entire detector is achieved by cross-interconnecting between the clusters (Fig. 2).

\subsection{Calibration system setup and test}

Take pool 1\# for example, the calibration system contains a 25-channel motherboard controlled by computer, provides LED control instructions and LED driver power supply through a multi-core cable; 25 4-channel LED drivers (2 channels are used); 25 LED light tubes, for each tube, one end 

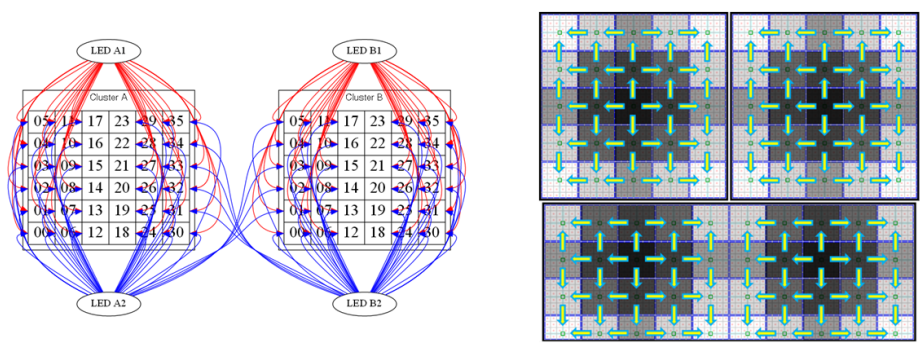

Figure 2: Cross calibration scheme.

connected to the LED driver channel with a $1.5 \mathrm{~m}$ long SMA coaxial cable and the other end is connected to a plastic fiber bundle with threaded connection.

The calibration system of pool 2\# is as same as pool 1\#, for pool 3\#, 2 mother boards and 40 LED drivers are applied due to detector layout.

Since the plastic fibers have cropping errors in the production process with an error of several cms, the relative time offsets among the fibers in the bundle need to be calibrated after the LED and the fiber bundle assembly. For this purpose, a test platform based on high-precision TDC, onedimensional electric slide and fast PMT for relative time offsets of fibers is built [2]. After the test, the time accuracy of optical fiber in calibration system is $10 \mathrm{ps}$, which can meet the requirements of calibration accuracy. The test results are used in the calibration system are shown in Fig. 3 and is deducted from the calibration data.

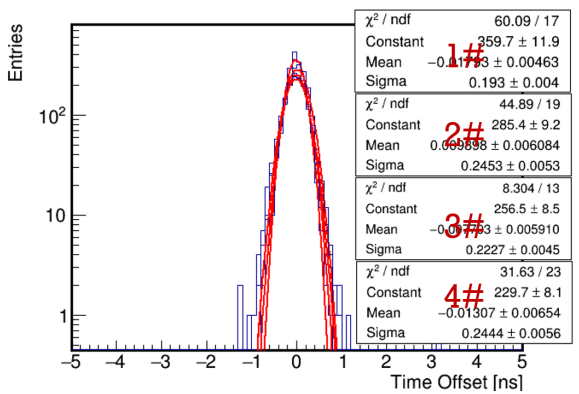

Figure 3: Fiber time offsets. 3\# 4\# means the west half and east half of pool 3\#.

\subsection{Calibration workflow and results}

To distinguish between normal physical data acquisition, the hardware time calibration runs separately for pools. For any pool, it takes about 30 minutes to calibrate one time, it includes the calibration system start-up and switching of operating mode, the running mode and running time information is recorded by the computer. To better distinguish the LED signals, the LED1 and LED2 are lit for 10 minutes, respectively, at a frequency of $4 \mathrm{kHz}$ to ensure that sufficient amount 
of data is obtained. Take pool 1\# for example, 25 LEDs are driven, and synchronized within $50 \mathrm{~ns}$, ensuring that all LED luminescent signals are recorded in the same data acquisition window.

After the data acquisition is completed, according to the log information recorded by the computer, the data of LED1 and LED2 signals are distinguished, Fig. 4 is the charge signal and time information recorded by PMT in a calibration run, and the LED signal of the calibration data is fitted to get the mean value of time and charge. Data process takes two steps:
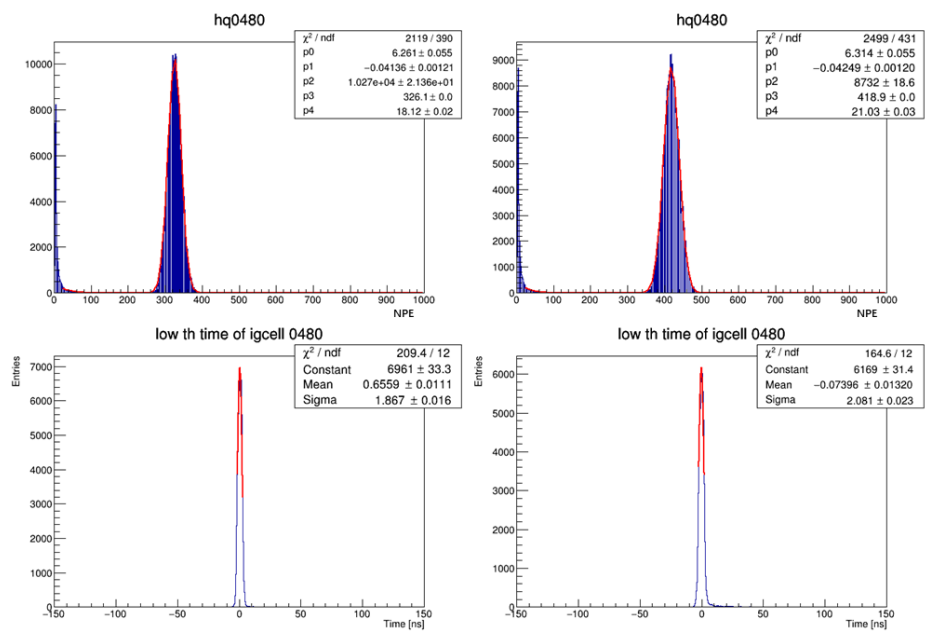

Figure 4: The LED signals for 1 PMT in a calibration run.

- Q-T correction

Fig. 5 shows the Q-T relationship of 20-inch PMT. The curve is fitted by a power-law function as below.

$$
T(Q)=p 0 \times Q^{p 1}+p 2
$$

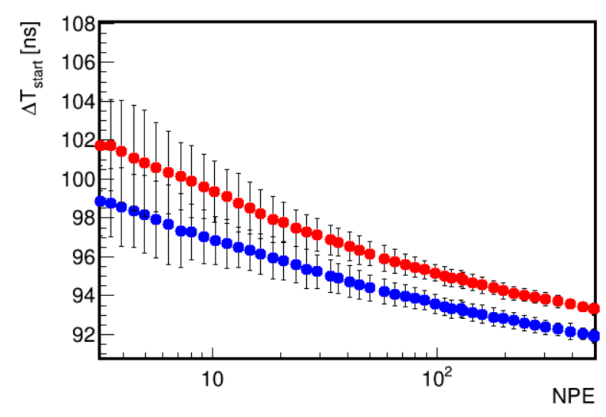

Figure 5: Q-T curve.

- Cross-calculation

As mentioned above, the entire detector is calibrated by cross fibers, the calculation method as below : 
choose $\mathrm{CH} 0$ as a reference channel in cluster $0, \mathrm{CHi}$ is in neighbouring cluster ( called clusteri ), the time offset $T_{\text {of } f \text { set }}$ is

$$
\begin{aligned}
& T_{\text {off } s e t \_} i=T_{c i \_c h i}-T_{c 0 \_c h 0}=\left(T A_{c i \_c h i}-T A_{c i \_c h x}\right)-\left(T B_{c i \_c h x}-T B_{c 0 \_c h 0}\right) \\
& T_{\text {of f set_ }} j=T_{c i_{-} c h i}-T_{c 0 \_c h 0}=\left(T B_{c i \_c h i}-T B_{c 0 \_} c h x\right)-\left(T A_{c 0 \_c h x}-T B_{c 0 \_c h 0}\right)
\end{aligned}
$$

in the equation,

* $T_{\text {of } f \text { set }} i$ is calculation result using cross channels in clusteri;

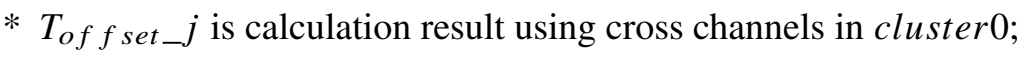

* TA $A_{c i}$ chi $i$ is LEDA data for channeli in clusteri;

* TA $A_{\text {ci_chx }}$ is LEDA data for channelx of clusteri (channelx is one of the cross channels in clusteri);

* TB $B_{\text {ci_chx }}$ is LEDB data for channelx of clusteri (channelx is one of the cross channels in clusteri);

* TB $B_{c 0 \_c h 0}$ is LEDB data for channel0 of cluster 0 ;

as it is described above, there are 8 cross channels in 2 neighbouring clusters, so there are 8 independent path to calculate the time offset, which can minimize the system error:

$$
<T_{\text {off set }}>=\frac{\sum_{i=1}^{4} T_{\text {offset_ }} i+\sum_{j=1}^{4} T_{\text {off } \text { set }_{-} j}}{8}
$$

For an entire pool, 4 pathways are designed to calculate the time offset between test cluster and the reference cluster, then take the average results of these pathways, also take pool 1\# as example, all the 25 cluster will be the reference cluster, 25 set of time offset results are obtained. All the results are averaged, in this way, the system error can be reduced, also the bad channel can be checked. The calibration results of pool 1\# and pool 2\# are shown in Fig. 6.

The hardware time calibration precision is evaluated as the distribution width of the difference of time offsets for all PMT channels, with different calibration run in a short time (eg. 2 or 3 days), as it is shown in Fig. 7, fitting with a Gaussian funtion, the precision equals $\sigma / \sqrt{2}$. It can be easily calculated that the precision of time calibration system is less than $40 \mathrm{ps}$.

The time offset among these pools is monitored through cosmic showers by now, another set of calibration module covers cells of 3 pools will be installed in the near future. Since pool 3\# is still is in the acceptance and debugging process, the results will not carried out this time.

\section{Offline time calibration using small shower events}

The calibrated time offsets from the hardware method can be used as the input of the event reconstruction. With the complicated models, the directions of the events could be reconstructed and these direction parameters could be used to fit the events. The reconstruction models could be used to predicate the arrival time of each cell, so that the residual time of each cell is got by 

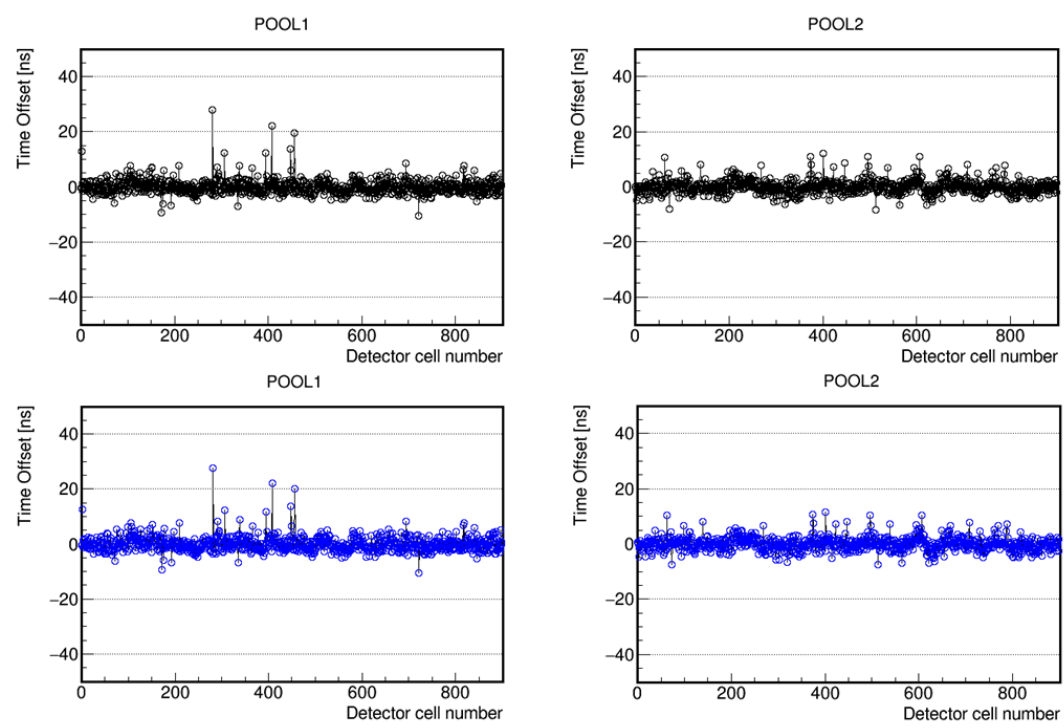

Figure 6: Calibration result of pool 1\# and pool 2\#. The black dot is low-threshold result and the blue dot is high-threshold result.
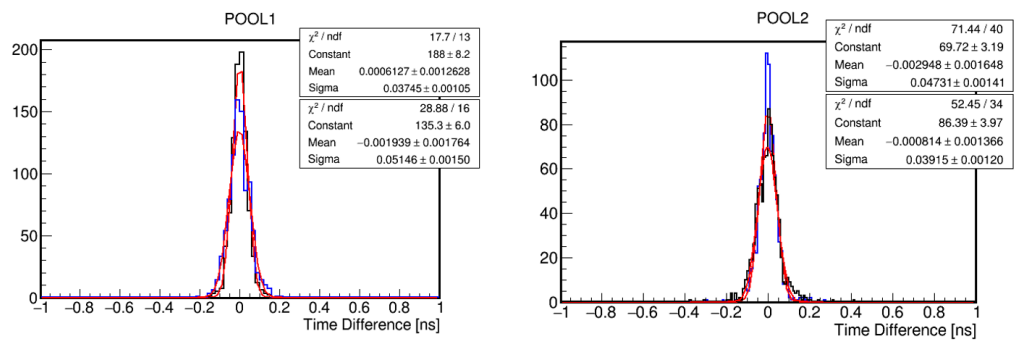

Figure 7: Calibration result of pool 1\# and pool 2\#. The black line is low-threshold result and the blue line is high-threshold result.

comparing with the real detected time. Assuming that there is a detector cell with a large time offset, then there will be a systematic offset for the residual time by using a lot of reconstructed events.

An offline time calibration has been developed in order to validate the time offsets calibrated by hardware method and reduce the residual time of all the detector cells. In order to simplify the reconstruction model using the plane shower fronts, small shower events are used, whose propagation could be described using a plane front. The detectors are divided into small clusters with 36 detector cells in each cluster. The calibrated time offsets of the full detectors are combined using these small clusters.

As shown in Fig. 8, the direction of a shower is $\mathbf{k}$. For each detector cell $i$, the predicated hit time $T_{i}^{\text {pred }}$ is described as following:

$$
T_{i}^{\text {pred }}=T_{c}+\mathbf{k} \cdot\left(\mathbf{X}_{i}-\mathbf{X}_{c}\right) / c
$$

A residual time $\delta_{i}$ is defined as 


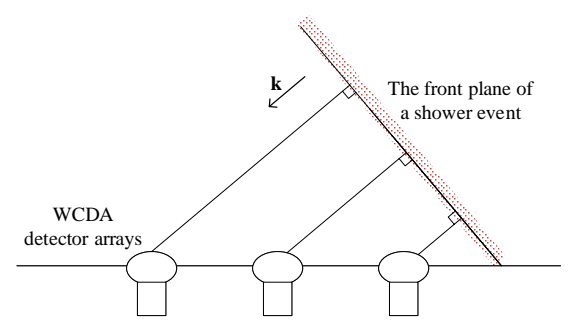

Figure 8: Schematic view of plane fit

$$
\delta_{i}=T_{i}^{\text {meas }}-T_{i}^{\text {pred }}
$$

The least square method is applied to get the parameter $\mathbf{k}$. The residual time could be then calculated using the fit parameters.

\subsection{Calibration of the pools}

As the small shower events are used, the selected detector regions for event reconstruction are limited to $10 \times 10$ detector cells, as shown in the Fig. 9. Only the $4 \times 4$ detector cells at the center of $10 \times 10$ detector cells are used for time calibration. Then the four $4 \times 4$ detector cells could be combined together into one cluster of $6 \times 6$ detector cells.

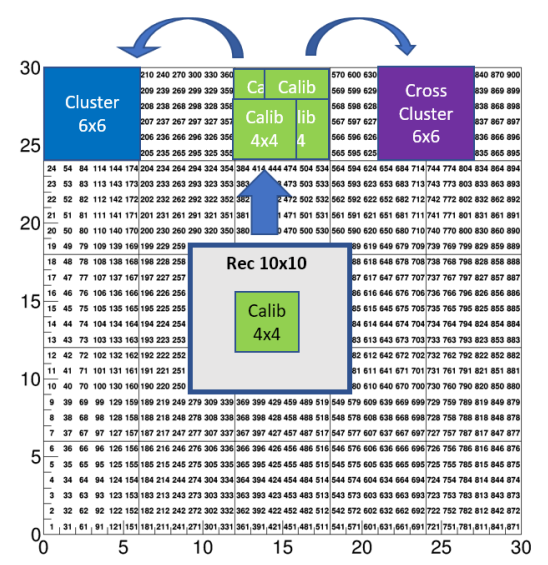

Figure 9: The schematic view of the full pool calibration

In order to combine the clusters with $6 \times 6$ detector cells into the full detectors, a "cross" cluster which overlaps with the two clusters are introduced. The center cluster has the highest priority which will update the four closest clusters. Each of the cluster will then update the closest lower priority clusters.

\subsection{Correction at the edge of pools}

The event reconstruction at the detector edge is limited due to missing part of cells in the $10 \times 10$ regions. In order to correct such edge effects, the $24 \times 24$ detectors are used to get the 


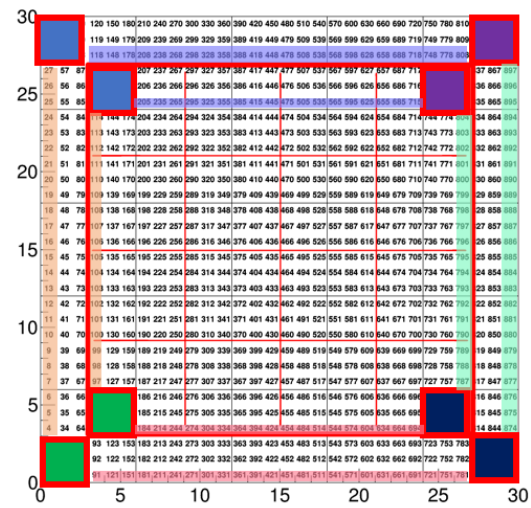

Figure 10: The schematic view of the edge correction

calibrated time offsets. The time offsets of the $24 \times 24$ detectors could be calibrated using the method described in the previous section.

As shown in Fig. 10, the edge regions are divided into several parts: the four corners, the three columns or rows. For an example, two mean values could be calculated for the left-top regions in blue. The difference between two values is used to correct the blue region in $30 \times 30$.

\section{Conclusion}

The time calibration is important since the time offsets between the PMTs are used in cosmic shower direction reconstruction, which determine the pointing error of the $\gamma$ ray sources. With the help of hardware calibration system, a high precision of calibration result is given and applied in the Crab observation [3]. With the offline calibration by cosmic shower event, the offsets between pools and cross check with hardware calibration result can be done. After pool 3\# debugging, the whole LHAASO-WCDA time calibration will be taken out.

\section{References}

[1] Cao Zhen, et al., Introduction to Large High Altitude Air Shower Observatory (LHAASO), Chinese Astronomy and Astrophysics Volume 43, Issue 4, October-December 2019, Pages 457-478

[2] J.Y. Liu, et al., Test bench for fibers of the LHAASO-WCDA time calibration system. 2017 JINST 12 P10021

[3] F. Aharonian, et al., Performance of LHAASO-WCDA and Observation of the Crab Nebula as a Standard Candle[J]. Chinese Physics C. 